\title{
On the Performance of Kernel Methods for Skin Color Segmentation
}

\author{
A. Guerrero-Curieses, ${ }^{1}$ J. L. Rojo-Álvarez, ${ }^{1}$ P. Conde-Pardo, ${ }^{2}$ I. Landesa-Vázquez, ${ }^{2}$ \\ J. Ramos-López, ${ }^{1}$ and J. L. Alba-Castro
}

${ }^{1}$ Departamento de Teoría de la Señal y Comunicaciones, Universidad Rey Juan Carlos, 28943 Fuenlabrada, Spain

${ }^{2}$ Departamento de Teoría de la Señal y Comunicaciones, Universidad de Vigo, 36200 Vigo, Spain

Correspondence should be addressed to A. Guerrero-Curieses, alicia.guerrero@urjc.es

Received 26 September 2008; Revised 23 March 2009; Accepted 7 May 2009

Recommended by C.-C. Kuo

\begin{abstract}
Human skin detection in color images is a key preprocessing stage in many image processing applications. Though kernel-based methods have been recently pointed out as advantageous for this setting, there is still few evidence on their actual superiority. Specifically, binary Support Vector Classifier (two-class SVM) and one-class Novelty Detection (SVND) have been only tested in some example images or in limited databases. We hypothesize that comparative performance evaluation on a representative application-oriented database will allow us to determine whether proposed kernel methods exhibit significant better performance than conventional skin segmentation methods. Two image databases were acquired for a webcam-based face recognition application, under controlled and uncontrolled lighting and background conditions. Three different chromaticity spaces (YCbCr, CIEL* $\mathrm{a}^{*} \mathrm{~b}^{*}$, and normalized RGB) were used to compare kernel methods (two-class SVM, SVND) with conventional algorithms (Gaussian Mixture Models and Neural Networks). Our results show that two-class SVM outperforms conventional classifiers and also one-class SVM (SVND) detectors, specially for uncontrolled lighting conditions, with an acceptably low complexity.
\end{abstract}

Copyright (C) 2009 A. Guerrero-Curieses et al. This is an open access article distributed under the Creative Commons Attribution License, which permits unrestricted use, distribution, and reproduction in any medium, provided the original work is properly cited.

\section{Introduction}

Skin detection is often the first step in many image processing man-machine applications, such as face detection $[1,2]$, gesture recognition [3], video surveillance [4], human video tracking [5], or adaptive video coding [6]. Although pixelwise skin color alone is not sufficient for segmenting human faces or hands, color segmentation for skin detection has been proven to be an effective preprocessing step for the subsequent processing analysis. The segmentation task in most of the skin detection literature is achieved by using simple thresholding [7], histogram analysis [8], single Gaussian distribution models [9], or Gaussian Mixture Models (GMM) [1, 10, 11]. The main drawbacks of the distribution-based parametric modeling techniques are, first, their strong dependence on the chosen color space and lighting conditions, and second, the need for selection of the appropriate model for statistical characterization of both the skin and the nonskin classes [12]. Even with an accurate estimation of the parameters in any density-based parametric models, the best detection rate in skin color segmentation cannot be ensured. When a nonparametric modeling is adopted instead, a relatively high number of samples is required for an accurate representation of skin and nonskin regions, like histograms [13] or Neural Networks (NN) [12].

Recently, the suitability of kernel methods has been pointed out as an alternative approach for skin segmentation in color spaces [14-17]. First, the Support Vector Machine (SVM) was proposed for classifying pixels into skin or nonskin samples, by stating the segmentation problem as a binary classification task [17], and later, some authors have proposed that the main interest in skin segmentation could be an adequate description of the domain that supports the skin pixels in the space color, rather than devoting effort to model the more heterogeneous nonskin 
class $[14,15]$. According to this hypothesis, one-class kernel algorithms, known in the kernel literature as Support Vector Novelty Detection (SVND) $[18,19]$, have been used for skin segmentation.

However, and to our best knowledge, few exhaustive performance comparison have been made to date for supporting a significant overperformance of kernel methods with respect to conventional skin segmentation algorithms. More, different merit figures have been used in different studies, and even contradictory conclusions have been obtained when comparing SVM skin detectors with conventional parametric detectors [16, 17]. Moreover, the advantage of focusing on determining the region that supports most of the skin pixels in SVND algorithms, rather than modeling skin and nonskin regions simultaneously (as done in GMM, NN, and SVM algorithms), has not been thoroughly tested [14, 15].

Therefore, we hypothesize that comparative performance evaluation on a database, with identical merit figures, will allow us to determine whether proposed kernel methods exhibit significantly better performance than conventional skin segmentation methods. For this purpose, two image databases have been acquired for a webcam based face recognition application, under controlled and uncontrolled lighting and background conditions. Three different chromaticity spaces (YCbCr, CIEL*a*b*, normalized RGB) are used to compare kernel methods (SVM and SVND) with conventional skin segmentation algorithms (GMM and $\mathrm{NN}$ ).

The scheme of this paper is as follows. In Section 2, we summarize the state of the art in skin color representation and segmentation, and we highlight some recent findings that explain the apparent lack of consensus on some issues regarding the optimum color spaces, fitting models, and kernel methods. Section 3 summarizes the wellknown GMM formulation, and presents a basic description of the kernel algorithms that are used here. In Section 4, performance is evaluated for conventional and for kernelbased segmentations, with emphasis on the free parameters tuning. Finally, Section 5 contains the conclusions of our study.

\section{Background on Color Skin Segmentation}

Pixelwise skin detection in color still images is usually accomplished in three steps: (i) color space transformation, (ii) parametric or nonparametric color distribution modeling, and (iii) binary skin/nonskin decision. We present the background on the main results in literature that are related to our work in terms of the skin pixels representation and of the kernel methods previously used in this setting.

2.1. Color Spaces and Distribution Modeling. The first step in skin segmentation, color space transformation, has been widely acknowledged as a necessary stage to deal with the perceptual nonparametricuniformity and with the high correlation among RGB channels, due to their mixing of luminance and chrominance information. However, although several color space transformations have been proposed and compared $[7,10,17,20]$, none of them can be considered as the optimal one. The selection of an adequate color space is largely dependent on factors like the robustness to changing illumination spectra, the selection of a suitable distribution model, and the memory or complexity constraints of the running application.

In the last years, experiments over highly representative datasets with uncontrolled lighting conditions have shown that the performance of the detector is degraded by those transformations which drop the luminance component. Also, color-distribution modeling has been shown to have a larger effect on performance than color space selection $[7,21]$. As trivially shown in [21], given an invertible oneto-one transformation between two $3 \mathrm{D}$ color spaces, if there exists an optimum skin detector in one space, there exists another optimum skin detector that performs exactly the same in the transformed space. Therefore, results of skin detection reported in literature for different color spaces must be understood as specific experiments constrained by the specific available data, the distribution model chosen to fit the specific transformed training data and the trainvalidationtest split to tune the detector.

Jayaram et al. [22] showed the performance of 9 color spaces with and without including the luminance component, on a large set of skin pixels under different illumination conditions from a face database, and nonskin pixels from a general database. With this experimental setup, histogram-based detection performed consistently better than Gaussian-based detection, both in 2D and in $3 \mathrm{D}$ spaces, whereas 3D detection performed consistently better than 2D detection for histograms but inconsistently better for Gaussian modeling. Also, regarding color space differences, some transformations performed better than RGB, but the differences were not statistically significant. Phung et al. [12] compared more distribution models (histogram-based, Gaussians, and GMM) and decisionbased classifiers (piecewise linear and NN) over 4 color spaces by using their ECU face and skin detection database. This database is composed of thousands of images with indoor and outdoor lighting conditions. The histogrambased Bayes and the MLP classifiers in RGB performed very similarly, and consistently better than the other Gaussianbased and piecewise linear classifiers. The performance over the four color spaces with high resolution histogram modeling was almost the same, as expected. Also, mean performance decreased and variance increased when the luminance component was discarded. In [17], the performance of nonparametric, semiparametric, and parametric approaches was evaluated over sixteen color spaces in $2 \mathrm{D}$ and $3 \mathrm{D}$, concluding that, in general, the performance does not improve with color space transformation, but instead it decreases with the absence of luminance. All these tests highlight the fact that with a rich representation of the $3 \mathrm{D}$ color space, color transformation is not useful at all but they bring also the lack of consensus regarding the performance of different color-distribution models, even when nonparametric ones seem to work better for large datasets. 
With these considerations in mind, and from our point of view, the design of the optimum skin detector for a specific application should consider the next situations.

(i) If there are enough labeled training data to generously fill the RGB space, at least the regions where the pixels of that application will map, and if RAM memory is not a limitation, a simple nonparametric histogram-based Bayes classifier over any color space will do the job.

(ii) If there is not enough RAM memory or enough labeled data to produce an accurate 3D-histogram, but still the samples represent skin under constrained lighting conditions, a chromaticity space with intensity normalization will probably generalize better when scarcity of data prevents modeling the 3D colorspace. The performance of any distributionbased or boundary-based classifier will be dependant on the training data and the colorspace, so a joint selection should end up with a skin detector that just works fine, but generalization could be compromised if conditions change largely.

(iii) If the spectral distribution of the prevailing light sources are heavily changing, unknown, or cannot be estimated or corrected, then better switch to another gray-based face detector because any try to build a skin detector with such a training set and conditions will yield unpredictable and poor results, unless dynamic adaptation of the skin color model in video sequences will be possible (see [23] for an example with known camera response under several color illuminants).

In this paper we study more deeply the second situation, that seems to be the most typical one for specific applications, and we will focus on the model selection for several $2 \mathrm{D}$ color spaces. We will analyze whether boundary-based models like kernel-methods work consistently better than distribution-based models, like classical GMM.

2.2. Kernel Methods for Skin Segmentation. The skin detection problem by using kernel-methods has been previously considered in literature. In [16] a comparative analysis of the performance of SVM on the features of a segmentation based on the Orthogonal Fourier-Mellin Moments can be found. They conclude that SVM achieves a higher face detection performance than a 3-layer Multilayer Perceptron (MLP) when an adequate kernel function and free parameters are used to train the SVM. The best tradeoff between the rate of correct face detection and the rate of correct rejection of distractors by using SVM is in the 65\%-75\% interval for different color spaces. Nevertheless, this database does not consider different illumination conditions. A more comprehensive review of color-based skin detection methods can be found in [17], which focus on classifying each pixel as skin or nonskin without considering any preprocessing stage. The classification performance, in terms of ROC (Receiver Operating Characteristic) curve and AUC (Area Under Curve), is evaluated by using SPM (Skin Probability
Map), GMM, SOM (Self-Organizing Map) and SVM on 16 color spaces and under varying lighting conditions. According to the results in terms of AUC, the best model is SPM, followed by GMM, SVM, and SOM. This is the only work where the performance obtained with kernelmethods is lower than that achieved with SPM and GMM. This work concludes that free parameter $v$ has little influence on the results, on the contrary to the rest of the works with kernel methods. Other works have shown that the histogram-based classifier can be an alternative to GMM [13] or even MLP [12] for skin segmentation problems. With our databases, the results obtained by the histogram-based method have not shown to be better than those from an MLP classifier.

These previous works have considered the skin detection as the skin/nonskin binary classification problem. Therefore, they used two-class kernel models. More recently, in order to avoid modeling nonskin regions, other approaches have been proposed to tackle the problem of skin detection by means of one-class kernel-methods. In [14], a one-class SVM model is used to separate face patterns from others. Although it is concluded that the extensive experiments show that this method has an encouraging performance, no further comparisons with other approaches are included, and few numerical results are reported. In [15], it is concluded that one-class kernel methods outperform other existing skin color models in normalized RGB and other color transformations, but again, comprehensive numerical comparisons are not reported, and no comparison, to other skin detectors are included.

Taking into account the previous works in literature, the superiority of kernel-methods to tackle the problem of skin detection should be shown by using an appropriate experimental setup and by making systematic comparisons with other models proposed to solve the problem.

\section{Segmentation Algorithms}

We next introduce the notation and briefly review the segmentation algorithms used in the context of skin segmentation applications, namely, the well-known GMM segmentation and the kernel methods with binary SVM and one-class SVND algorithms.

3.1. GMM Skin Segmentation. GMM for skin segmentation $[11,13]$ can be briefly described as follows. The a priori probability $P(\mathbf{x}, \Theta)$ of each skin color pixel $\mathbf{x}$ (in our case, $\mathbf{x} \in$ $\mathbb{R}^{2}$; see Section 4) is assumed to be the weighted contribution of $k$ Gaussian components, each being defined by parameter vector $\boldsymbol{\theta}_{i}=\left\{w_{i}, \boldsymbol{\mu}_{i}, \boldsymbol{\Sigma}_{i}\right\}$, where $w_{i}$ is the weight value of the $i$ th component, and $\boldsymbol{\mu}_{i}, \boldsymbol{\Sigma}_{i}$, are its mean vector and covariance matrix, respectively. The whole set of free parameters will be denoted by $\Theta=\left\{\boldsymbol{\theta}_{1}, \ldots, \boldsymbol{\theta}_{K}\right\}$. Within a Bayesian approach, the probability for a given color pixel $\mathbf{x}$ can be written as

$$
P(\mathbf{x}, \Theta)=\sum_{i=1}^{k} w_{i} p(\mathbf{x} i)
$$


where the ith component is given by

$$
p(x \mid i)=\frac{1}{(2 \pi)^{d / 2}\left|\boldsymbol{\Sigma}_{i}\right|^{1 / 2}} e^{-1 / 2\left(\mathbf{x}-\mu_{i}\right)^{T} \boldsymbol{\Sigma}_{i}^{-1}\left(\mathbf{x}-\boldsymbol{\mu}_{i}\right)},
$$

and the relative weights $w_{i}$ fulfill $\sum_{i=1}^{k} w_{i}=1$ and $w_{i} \geq 0$. Adjustable free parameters $\Theta$ are estimated by minimizing the negative log-likelihood for a training dataset, given by $\mathfrak{X} \equiv\left\{\mathbf{x}_{1}, \ldots, \mathbf{x}_{l}\right\}$, that is, we minimize

$$
-\ln \prod_{j=1}^{l} P\left(\mathbf{x}_{j}, \Theta\right)=-\sum_{j=1}^{l} \ln \sum_{i=1}^{k} w_{i} p\left(\mathbf{x}_{j} i\right) .
$$

The optimization is addressed by using the EM algorithm [24], which calculates the a posteriori probabilities as

$$
P^{t}\left(i \mathbf{x}_{j}\right)=\frac{w_{i}^{t} p^{t}\left(\mathbf{x}_{j} i\right)}{P^{t}\left(\mathbf{x}_{j}, \Theta\right)},
$$

where superscript $t$ denotes the parameter values at $t$ th iteration. The new parameters are obtained by

$$
\begin{aligned}
\boldsymbol{\mu}_{i}^{t+1} & =\frac{\sum_{j=1}^{l} P^{t}\left(i \mid \mathbf{x}_{j}\right) \mathbf{x}_{j}}{\sum_{j=1}^{l} P^{t}\left(i \mid \mathbf{x}_{j}\right)}, \\
\boldsymbol{\Sigma}_{i}^{t+1} & =\frac{\sum_{j=1}^{l} P^{t}\left(i \mid \mathbf{x}_{j}\right)\left(\mathbf{x}-\boldsymbol{\mu}_{i}\right)^{T}\left(\mathbf{x}-\boldsymbol{\mu}_{i}\right)}{\sum_{j=1}^{l} P^{t}\left(i \mid \mathbf{x}_{j}\right)}, \\
w_{i}^{t+1} & =\frac{1}{l} \sum_{j=1}^{l} P^{t}\left(i \mid \mathbf{x}_{j}\right) .
\end{aligned}
$$

The final model will depend on model order $K$, which has to be analyzed in each particular problem for the best biasvariance tradeoff.

A $k$-means algorithm is often used, in order to take into account even poorly represented groups of samples. All components are initialized to $w_{i}=1 / k$ and the covariance matrices $\boldsymbol{\Sigma}_{i}$ to $\delta^{2} \mathbf{I}$, where $\delta$ is the Euclidean distance from the component mean $\boldsymbol{\mu}_{i}$ of the nearest neighbor.

3.2. Kernel-Based Binary Skin Segmentation. Kernel methods provide us with efficient nonlinear algorithms by following two conceptual steps: first, the samples in the input space are nonlinearly mapped to a high-dimensional space, known as feature space, and second, the linear equations of the data model are stated in that feature space, rather than in the input space. This methodology yields compact algorithm formulations, and leads to single-minimum quadratic programming problems when nonlinearity is addressed by means of the socalled Mercer's kernels [25].

Assume that $\left\{\left(\mathbf{x}_{i}, y_{i}\right)\right\}_{i=1}^{l}$, with $\mathbf{x}_{i} \in \mathbb{R}^{2}$, represents a set of $l$ observed skin and nonskin samples in a space color, with class labels $y_{i} \in\{-1,1\}$. Let $\varphi: \mathbb{R}^{2} \rightarrow F$ be a possibly nonlinear mapping from the color space to a possibly higherdimensional feature space $F$, such that the dot product between two vectors in $F$ can be readily computed using a bivariate function $K(\mathbf{x}, \mathbf{y})$, known as Mercer's kernel, that fulfills Mercer's theorem [26], that is,

$$
K(\mathbf{x}, \mathbf{y})=\langle\varphi(\mathbf{x}), \varphi(\mathbf{y})\rangle
$$

For instance, a Gaussian kernel is often used in support to vector algorithms, given by

$$
K(\mathbf{x}, \mathbf{y})=e^{-\|\mathbf{x}-\mathbf{y}\|^{2} / 2 \sigma^{2}}
$$

where $\sigma$ is the kernel-free parameter, which must be previously chosen, according to some criteria about the problem at hand and the available data. Note that, by using Mercer's kernels, nonparametriclinear mapping $\varphi$ does not need to be explicitly known.

In the most general case of nonparametriclinearly separable data, the optimization criterion for the binary SVM consists of minimizing

$$
\frac{1}{2}\|\mathbf{w}\|^{2}+C \sum_{i=1}^{l} \xi_{i}
$$

constrained to $y_{i}\left(\left\langle\mathbf{w}, \varphi\left(\mathbf{x}_{i}\right)\right\rangle+b\right) \geq 1-\xi_{i}$ and to $\xi_{i} \geq 0$, for $i=1, \ldots, l$. Parameter $C$ is introduced to control the tradeoff between the margin and the losses. By using the Lagrange Theorem, the Lagrangian functional can be stated as

$$
\begin{aligned}
L_{\mathrm{pd}}= & \frac{1}{2}\|\mathbf{w}\|^{2}+C \sum_{i=1}^{l} \xi_{i}-\sum_{i=1}^{l} \beta_{i} \xi_{i} \\
& -\sum_{i=1}^{l} \alpha_{i}\left(\left(y_{i}\left\langle\mathbf{w}, \varphi\left(\mathbf{x}_{i}\right)\right\rangle+b\right)-1+\xi_{i}\right)
\end{aligned}
$$

constrained to $\alpha_{i}, \beta_{i} \geq 0$, and it has to be maximized with respect to dual variables $\alpha_{i}, \beta_{i}$ and minimized with respect to primal variables $\mathbf{w}, b, \xi_{i}$. By taking the first derivative with respect to primal variables; the Karush-Khun-Tucker (KKT) conditions are obtained, where

$$
\mathbf{w}=\sum_{i=1}^{l} \alpha_{i} \varphi\left(\mathbf{x}_{i}\right),
$$

and the solution is achieved by maximizing the dual functional:

$$
\sum_{i=1}^{l} \alpha_{i}-\frac{1}{2} \sum_{i, j=1}^{l} \alpha_{i} \alpha_{j} y_{i} y_{j} K\left(\mathbf{x}_{i}, \mathbf{x}_{j}\right),
$$

constrained to $\alpha_{i} \geq 0$ and $\sum_{i=1}^{l} \alpha_{i} y_{i}=0$. Solving this quadratic programming (QP) problem yields Lagrange multipliers $\alpha_{i}$, and the decision function can be computed as

$$
f(\mathbf{x})=\operatorname{sgn}\left(\sum_{i=1}^{l} \alpha_{i} y_{i} K\left(\mathbf{x}, \mathbf{x}_{i}\right)+b\right)
$$

which has been readily expressed in terms of Mercer's kernels in order to avoid the explicit knowledge of the feature space and of the nonlinear mapping $\varphi$, and where $\operatorname{sgn}()$ denotes the sign function for a real number. 


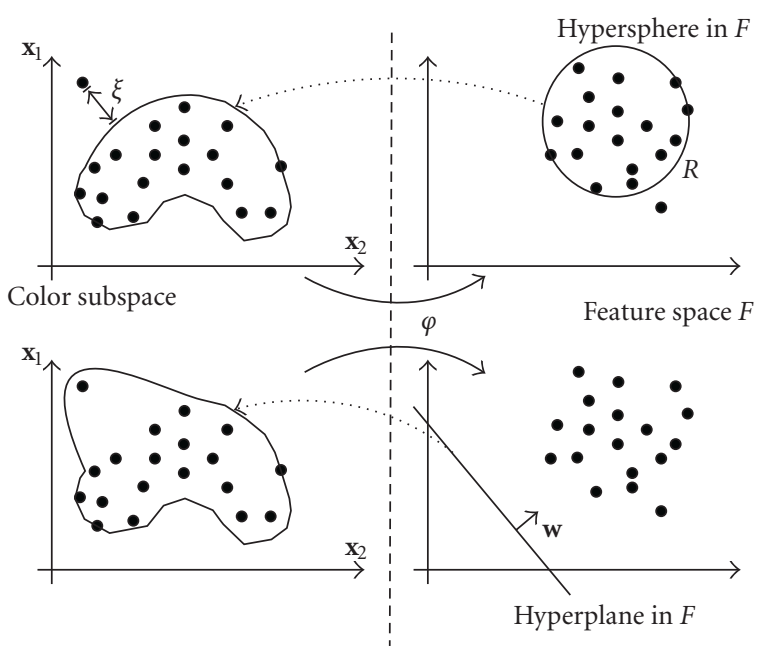

FIGURE 1: SVND algorithms make a nonlinear mapping from the input space to the feature space. A simple geometric figure (hypersphere or hyperplane) is traced therein, which splits the feature space into known domain and unknown domain. This corresponds to a nonlinear, complex geometry boundary in the input space.

Note from (10) that hyperplane in $F$ is given by a linear combination of the mapped input vectors, and accordingly, the patterns with $\alpha_{i} \neq 0$ are called Support Vectors. They contain all the relevant information for describing the hyperplane in $F$ that separates the data in the input space. The number of support vector is usually small (i.e, SVM gives a sparse solution), and it is related to the generalization error of the classifier.

3.3. Kernel-Based One-Class Skin Segmentation. The domain description of a multidimensional distribution can be addressed by using kernel algorithms that systematically enclose the data points into a nonlinear boundary in the input space. SVND algorithms distinguish between the class of objects represented in the training set and all the other possible objects. It is important to highlight that SVND represents a very different problem than the SVM. The training of SVND only uses training samples from one single class (skin pixels), whereas an SVM approach requires training with pixels from two different classes (skin and nonskin). Hence, let $\mathfrak{X} \equiv\left\{\mathbf{x}_{1}, \ldots, \mathbf{x}_{l}\right\}$ be now a set of $l$ observed only skin samples in a space color. Note that, in this case, nonskin samples are not used in the training dataset.

Two main algorithms for SVND have been proposed, that are based on different geometrical models in the feature space, and their schematic is depicted in Figure 1. One of them uses a maximum margin hyperplane in $F$ that separates the mapped data from the origin of $F$ [18], whereas the other finds a hypersphere in $F$ with minimum radius enclosing the mapped data [19]. These algorithms are next summarized.

3.3.1. SVND with Hyperplane. The SVND algorithm proposed in [18] builds a domain function whose value is +1 in the half region of $F$ that captures most of the data points, and -1 in the other half region. The criterion followed therein consists of first mapping the data into $F$, and then separating the mapped points from the origin with maximum margin. This decision function is required to be positive for most training vectors $\mathbf{x}_{i}$, and it is given by

$$
f(\mathbf{x})=\operatorname{sgn}(\langle\mathbf{w}, \boldsymbol{\varphi}(\mathbf{x})\rangle-\rho),
$$

where $\mathbf{w}, \rho$, are the maximum margin hyperplane and the bias, respectively. For a newly tested point $\mathbf{x}$, decision value $f(\mathbf{x})$ is determined by mapping this point to $F$ and then evaluating to which side of the hyperplane it is mapped.

In order to state the problem, two terms are simultaneously considered. On the one hand, the maximum margin condition can be introduced as usual in SVM classification formulation [26], and then, maximizing the margin is equivalent to minimizing the norm of the hyperplane vector w. On the other hand, the domain description is required to bound the space region that contains most of the observed data, but slack variables $\xi_{i}$ are introduced in order to consider some losses, that is, to allow a reduced number of exceptional samples outside the domain description. Therefore, the optimization criterion can be expressed as the simultaneous minimization of these two terms, that is, we want to minimize

$$
\frac{1}{2}\|\mathbf{w}\|^{2}+\frac{1}{v l} \sum_{i=1}^{l} \xi_{i}-\rho,
$$

with respect to $\mathbf{w}, \rho$ and constrained to

$$
\left\langle\mathbf{w}, \varphi\left(\mathbf{x}_{i}\right)\right\rangle \geq \rho-\xi_{i},
$$

and to $\rho>0$, and to $\xi_{i} \geq 0$, for $i=1, \ldots, l$. Parameter $\nu \in(0,1)$ is introduced to control the tradeoff between the margin and the losses.

The Lagrangian functional can be stated, similarly to the preceding subsection, and now, the dual problem reduces to minimizing

$$
\frac{1}{2} \sum_{i, j=1}^{l} \alpha_{i} \alpha_{j} K\left(\mathbf{x}_{i}, \mathbf{x}_{j}\right)
$$

constrained to the KKT conditions given by $\sum_{i=1}^{l} \alpha_{i}=1,0 \leq$ $\alpha_{i} \leq 1 / \nu l$, and $\mathbf{w}=\sum_{i=1}^{l} \alpha_{i} \varphi\left(\mathbf{x}_{i}\right)$.

It can be easily shown that samples $\mathbf{x}_{i}$ that are mapped into the +1 semispace have no losses $\left(\xi_{i}=0\right)$ and a null coefficient $\alpha_{i}$, so that they are not support vectors. Also, the samples $\mathbf{x}_{i}$ that are mapped to the boundary have no losses, but they are support vectors with $0<\alpha_{i}<1 / \nu l$, and accordingly they are called unbounded support vectors. Finally, samples $\mathbf{x}_{i}$ that are mapped outside the domain region have nonzero losses, $\xi_{i}>0$, their corresponding Lagrange multipliers are $\alpha_{i}=1 / \nu l$, and they are called bounded support vectors.

Solving this QP problem, the decision function (13) can be easily rewritten as

$$
f(\mathbf{x})=\operatorname{sgn}\left(\sum_{i=1}^{l} \alpha_{i} K\left(\mathbf{x}, \mathbf{x}_{i}\right)-\rho\right) .
$$


By now inspecting the KKT conditions, we can see that, for $v$ close to 1 , the solution consists of all $\alpha_{i}$ being at the (small) upper bound, which closely corresponds to a thresholded Parzen window nonparametric estimator of the density function of the data. However, for $v$ close to 0 , the upper boundary of the Lagrange multipliers increases and more support vectors become then unbounded, so that they are model weights that are adjusted for estimating the domain that supports most of the data.

Bias value $\rho$ can be recovered noting that any unbounded support vector $\mathbf{x}_{j}$ has zero losses, and then it fulfills.

$$
\sum_{i=1}^{l} \alpha_{i} K\left(\mathbf{x}_{j}, \mathbf{x}_{i}\right)-\rho=0 \Longrightarrow \rho=\sum_{i=1}^{l} \alpha_{i} K\left(\mathbf{x}_{j}, \mathbf{x}_{i}\right) .
$$

It is convenient to average the value of $\rho$ that is estimated from all the unbounded support vectors, in order to reduce the round-off error due to the tolerances of the QP solver algorithm.

3.3.2. SVND with Hypersphere. The SVND algorithm proposed in [19] follows an alternative geometric description of the data domain. After the input training data are mapped to feature space $F$, the smallest sphere of radius $R$, centered at $\mathbf{a} \in F$, is built under the condition that encloses most of the mapped data inside it. Soft constrains can be considered by introducing slack variables or losses, $\xi_{i} \geq 0$, in order to allow a small number of atypical samples being outside the domain sphere. Then the primal problem can be stated as the minimization of

$$
R^{2}+C \sum_{i=1}^{l} \xi_{i}
$$

constrained to $\left\|\boldsymbol{\varphi}\left(\mathbf{x}_{i}\right)-\mathbf{a}\right\|^{2} \leq R^{2}+\xi_{i}$ for $i=1, \ldots, l$, where $C$ is now the tradeoff parameter between radius and losses.

Similarly to the preceding subsections, by using the Lagrange Theorem, the dual problem consists now of maximizing

$$
-\sum_{i, j=1}^{l} \alpha_{j} \alpha_{i} K\left(\mathbf{x}_{j}, \mathbf{x}_{i}\right)+\sum_{i=1}^{l} \alpha_{i} K\left(\mathbf{x}_{i}, \mathbf{x}_{i}\right)
$$

constrained to the KKT conditions, and where the $\alpha_{i}$ are now the Lagrange multipliers corresponding to the constrains.

The KKT conditions allow us to obtain the sphere center in the feature space, $\mathbf{a}=\sum_{i=1}^{l} \alpha_{i} \varphi\left(\mathbf{x}_{i}\right)$, and then, the distance of the image of a given point $\mathbf{x}$ to the center can be calculated as

$$
\begin{aligned}
D^{2}(\mathbf{x})= & \|\boldsymbol{\varphi}(\mathbf{x})-\mathbf{a}\|^{2}=K(\mathbf{x}, \mathbf{x}) \\
& -2 \sum_{i=1}^{l} \alpha_{i} K\left(\mathbf{x}_{i}, \mathbf{x}\right)+\sum_{i, j=1}^{l} \alpha_{i} \alpha_{j} K\left(\mathbf{x}_{i}, \mathbf{x}_{j}\right) .
\end{aligned}
$$

In this case, samples $\mathbf{x}_{i}$ that are mapped strictly inside the sphere have no losses and null coefficient $\alpha_{i}$, and are not support vectors. Samples $\mathbf{x}_{i}$ that are mapped to the sphere boundary have no losses, and they are support vectors with $0<\alpha_{i}<C$ (unbounded support vectors). Samples $\mathbf{x}_{i}$ that are mapped outside the sphere have nonzero losses, $\xi_{i}>0$, and their corresponding Lagrange multipliers are $\alpha_{i}=C$ (bounded support vectors). Therefore, the radius of the sphere is the distance to the center in the feature space, $D\left(\mathbf{x}_{j}\right)$, for any support vector $\mathbf{x}_{j}$ whose Lagrange multiplier is different from 0 and from $C$, that is, if we denote by $R_{0}$ the radius of the solution sphere, then

$$
R_{0}^{2}=D^{2}\left(\mathbf{x}_{\mathbf{j}}\right)
$$

The decision function for a new sample belonging to the domain region is now given by

$$
f(\mathbf{x})=\operatorname{sgn}\left(D^{2}(\mathbf{x})-R_{0}^{2}\right),
$$

which can be interpreted in a similar way to the SVND with hyperplane. A difference now is that a lower value of the decision statistic (distance to the hypersphere center) is associated with the skin domain, whereas in SVND with hyperplane, a higher value for the statistic (distance to the coordenate hyperorigin) is associated with the skin domain.

\section{Experiments and Results}

In this section, experiments are presented in order to determine the accuracy of conventional and kernel methods for skin segmentation. According to our application constraints, the experimental setting considered two main characteristics of the data, namely, the importance of controlled lighting and acquisition conditions, which was taken into account by using two different databases described next, and the consideration of three different chromaticity color spaces. In these situations, we analyzed the performance of two conventional skin detectors (GMM and MLP), and three kernel methods (binary SVM, and one-class hyperplane and hypersphere SVND algorithms).

4.1. Experiments and Results. As pointed out in Section 2, one of the main aspects to consider in the design of the optimum skin detector for a specific application is the lighting conditions. If lighting conditions (mainly its spectral distribution) can be controlled, a chromaticity space with intensity normalization will probably generalize better than a 3D one when there is not enough variability to represent the 3D color space. In order to tackle this problem, we will consider a database of face images in an office environment, acquired with several different webcams, with the goal of building a face recognition application for Internet services. With this setup, our restrictions are; (i) mainly Caucasian people considered; (ii) a mediumsize labeled dataset available; (iii) office background and mainly indoor lighting will be present (iv) webcams using the automatic white balance correction (control of color spectral distribution).

Databases. We considered using other available databases, for instance, XM2VTS database [27] for controlled lighting 


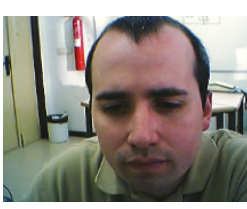

$(\mathrm{a} 0)$

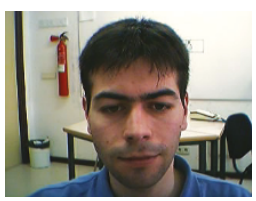

(b0)

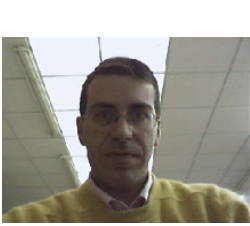

$(\mathrm{c} 0)$

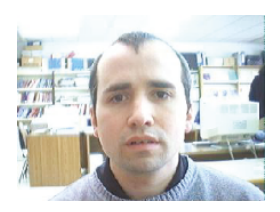

(d0)

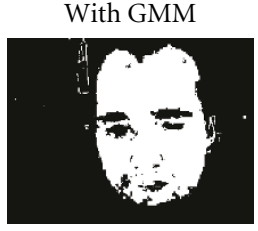

(a1)

With GMM

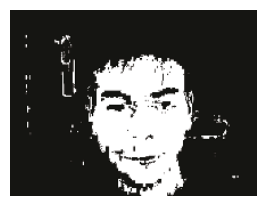

(b1)

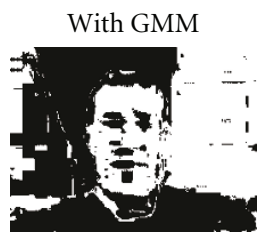

(c1)

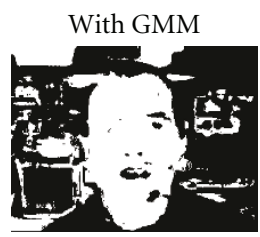

(d1)

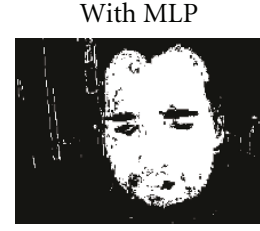

(a2)

With MLP

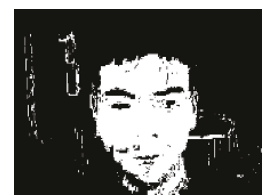

(b2)

With MLP

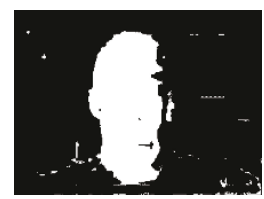

(c2)

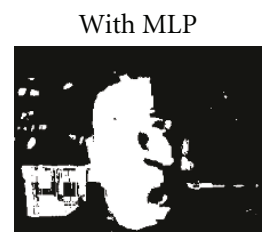

(d2)

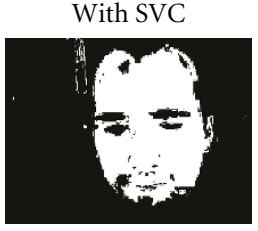

(a3)

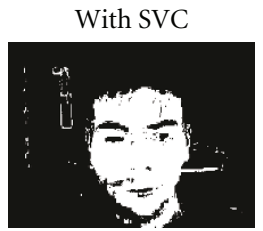

(b3)

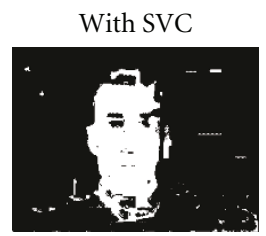

(c3)

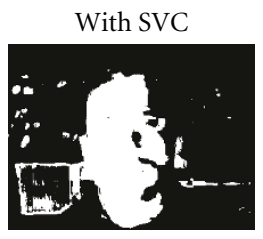

(d3)
With SVND-S

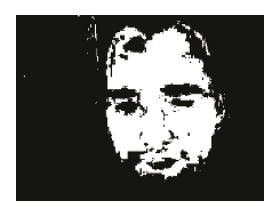

(a4)

With SVND-S

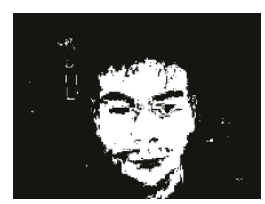

(b4)

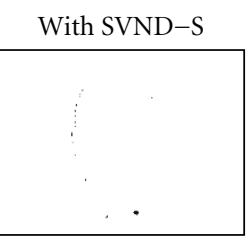

(c4)

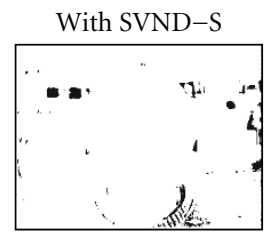

(d4)

FIGURE 2: Examples of RGB images in the databases: $(a 0, b 0)$ from CdB, and $(c 0, d 0)$ from UdB. Classifiers correspond to GMM $(* 1)$, MLP $(* 2)$, SVM $(* 3)$, and SVND-S $(* 4)$. Nonskin pixels in black and skin pixels in white.

and background conditions dataset, but color was poorly represented in these images due to video color compression. With BANCA [28] for uncontrolled lighting and background conditions dataset, we found the same restrictions. Therefore, we assembled our own databases.

First, a controlled dataBase (from now, CdB) of 224 face images from 43 different Caucasian people (examples in Figure $2(\mathrm{a} 0, \mathrm{~b} 0)$ ) was assembled. Images were acquired by the same webcam in the same place under controlled lighting conditions. The webcam was configured to output linear RGB with 8 bits per channel in snapshot mode. This database was used to evaluate the segmentation performance under controlled and uniform conditions.

Second, an uncontrolled dataBase (from now, UdB) of 129 face images from 13 different Caucasian people (examples in Figure $2(\mathrm{c} 0, \mathrm{~d} 0)$ ) was assembled. Images were taken from eight different webcams in automatic white balance configuration, in manual or automatic gain control, and under differently mixed lighting sources (tungsten, fluorescent, daylight). This database was used to evaluate the robustness of the detection methods under uncontrolled light intensity but similar spectral distribution.
For both databases, around half million skin and nonskin pixels were selected manually from RGB images.

Color Spaces. The pixels in the databases were subsequently labeled and transformed into the next color spaces.

(i) YCbCr, a color-difference coding space defined for digital video by the ITU. We used the recommendation ITU-R BT.601-4, that can be easily computed as an offset linear transformation of RGB.

(ii) CIEL* $a^{*} b^{*}$, a colorimetric and perceptually uniform color space defined by the Commission Internationale de L'Eclairage, nonlinearly and quite complexly related to $\mathrm{RGB}$.

(iii) normalized RGB, an easy nonparametriclinear transformation of RGB that normalizes every RGB channel by their sum, so that $r+g+b=1$.

Chrominance components of skin color in these spaces were assumed to be only slightly dependent on the luminance component (decreasingly dependent in $\mathrm{YCbCr}, \mathrm{CIEL}^{*} \mathrm{a}^{*} \mathrm{~b} *$, and normalized RGB) $[29,30]$. Hence, in order to reduce 


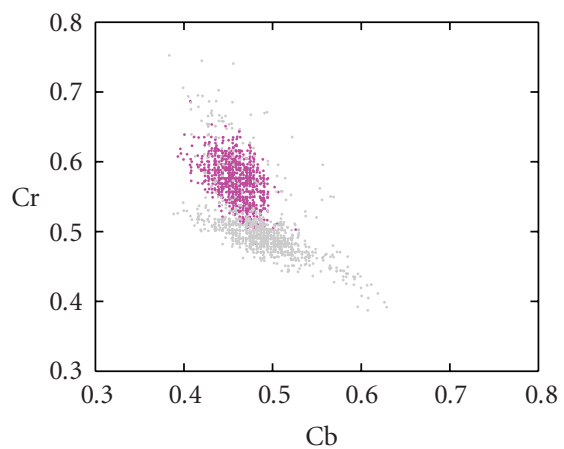

(a)

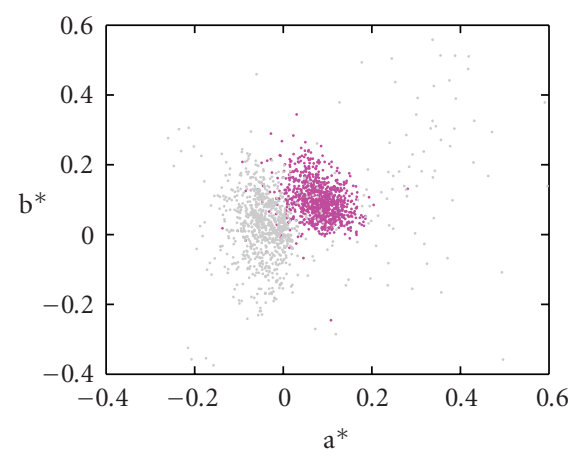

(b)

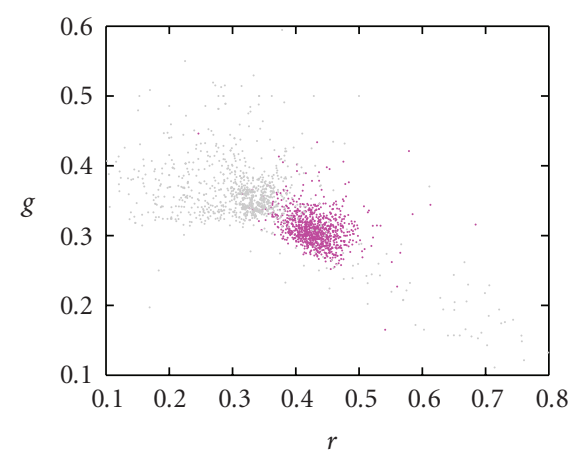

(c)

FIgure 3: CdB skin (red) and nonskin (gray) samples used for test: (a) in CbCr space; (b) in a*b* components CIEL*a*b* space; (c) in rg component from normalized RGB.

domain and distribution dimensionality, only 2D spaces were considered, and they were $\mathrm{CbCr}$ components in $\mathrm{YCbCr}$, $a^{*} b^{*}$ components in CIEL* $a^{*} b^{*}$, and $\mathrm{rg}$ components in normalized RGB. Figure 3 shows the resulting data for pixels in $\mathrm{CdB}$.

4.2. Experiments and Results. For each segmentation procedure, the Half Total Error Rate (HTER) was measured for featuring the performance provided by the method, that is,

$$
\mathrm{HTER}=\frac{\mathrm{FAR}+\mathrm{FRR}}{2} \times 100
$$

where FAR and FRR are False Acceptance and False Rejection Ratios, respectively, measured at the Equal Error Rate (EER) point, that is, in the point where the proportion of false acceptances is equal to the proportion of false rejections. Usually, the performance of a system is given over a test set and the working point is chosen over the training set. In this work we give the FAR, FRR and HTER figures for a system working in the EER point set in training.

The model complexity (MC) was also obtained as a figure of merit for the segmentation method, given by the number of Gaussian components in GMM, by the number of neurons in the hidden layer in MLP, and by the percentage of support vectors in kernel-based detectors, that is, $\mathrm{MC}=\# \mathrm{sv} / l \times 100$, where \#sv is the number of support vectors $\left(\alpha_{i}>0\right)$ and $l$ is the number of training samples.

The tuning set for adjusting the decision threshold consisted of the skin samples and the same amount of nonskin samples. Performance was evaluated in a disjoint set (test set) which included labeled skin and nonskin pixels.

4.3. Results with Conventional Segmentation. We used GMM as the base procedure to compare with due to it has been commonly used in color image processing for skin applications. Here, we used 90000 skin samples to train the model, 180000 non-skin and skin samples (the previous 90000 skin samples plus other 90000 non-skin samples) to adjust the threshold value, and new 250000 samples (170 000 of nonskin and 80000 of skin) to test the model.
TABLE 1: HTER values for GMM at EER working point with increasing number of mixtures.

\begin{tabular}{ccccccc}
\hline & & & \multicolumn{5}{c}{$k$} \\
& & $\mathbf{1}$ & $\mathbf{3}$ & $\mathbf{5}$ & $\mathbf{7}$ & $\mathbf{9}$ \\
\hline \multirow{4}{*}{$\mathrm{CdB}$} & $\mathbf{C b C r}$ & 11.5 & 11.9 & 12.9 & 12.8 & 12.9 \\
& $\mathbf{a}^{*} \mathbf{b}^{*}$ & 7.5 & 8.6 & 8.7 & 8.7 & 9.0 \\
& $\mathbf{r g}$ & 7.3 & 7.8 & 7.7 & 9.0 & 8.1 \\
\hline \multirow{4}{*}{$\mathrm{UdB}$} & $\mathbf{C b C r}$ & 24.1 & 25.6 & 25.1 & 25.5 & 23.9 \\
& $\mathbf{a}^{*} \mathbf{b}^{*}$ & 23.6 & 26.1 & 22.3 & 24.0 & 24.5 \\
& $\mathbf{r g}$ & 22.8 & 25.5 & 21.5 & 22.8 & 23.3 \\
\hline
\end{tabular}

Table 1 shows the HTER values for the three color spaces and the two databases considered with different number of Gaussian components (i.e, the model order) for the GMM model. The model with a single Gaussian yielded the minimum average error in segmentation when images were taken under controlled lighting conditions (CbB), but under uncontrolled lighting conditions (UdB) the optimum number of Gaussians was quite noisy for our dataset. As could be expected, results were better for pixel classification under controlled lighting conditions, below $12 \%$ of HTER in all model orders. Performance decreased under uncontrolled lighting conditions, showing values of HTER over $20 \%$ in the three color spaces.

Table 2 shows the results for GMM trained with different number of skin samples. In both databases (controlled and uncontrolled acquisition conditions) the performance in $\mathrm{CbCr}, \mathrm{a}^{\star} \mathrm{b}^{\star}$ and $\mathrm{rg}$ color spaces is similar. Nevertheless, performance for $\mathrm{UdB}$ was worse than for $\mathrm{CdB}$. It can be seen that under controlled acquisition conditions the results obtained for the three color spaces showed the lowest HTER for $=1$. Therefore, under controlled image capturing conditions, there was no apparent gain in using a more sophisticated model, and this result is coherent with the reported in [2]. By the values obtained for GMM under uncontrolled acquisition conditions, we can conclude that there is not a fix value of $k$ which offers statistically significant better results. 
TABLE 2: HTER values for GMM at EER working point with different number of skin training samples.

\begin{tabular}{cccccccc}
\hline & & \multicolumn{2}{c}{ GMM } & \multicolumn{2}{c}{ GMM } \\
& & \multicolumn{2}{c}{ 250 samples } & \multicolumn{2}{c}{ 90000 samples } \\
& & FAR-FRR & HTER & $k$ & FAR-FRR & HTER & $k$ \\
\hline \multirow{4}{*}{ CdB } & CbCr & $7.8-14.7$ & 11.3 & 1 & $12.0-11.0$ & 11.5 & 1 \\
& $\mathbf{a}^{*} \mathbf{b}^{*}$ & $4.2-10.0$ & 7.1 & 1 & $7.5-7.4$ & 7.5 & 1 \\
& $\mathbf{r g}$ & $5.9-8.8$ & 7.4 & 1 & $7.3-7.4$ & 7.3 & 1 \\
\hline \multirow{3}{*}{ UdB } & $\mathbf{C b C r}$ & $18.1-29.0$ & 23.6 & 1 & $24.0-23.8$ & 23.9 & 9 \\
& $\mathbf{a}^{*} \mathbf{b}^{*}$ & $17.9-27.2$ & 22.6 & 7 & $22.5-22.2$ & 22.3 & 5 \\
& $\mathbf{r g}$ & $21.9-21.8$ & 21.8 & 1 & $21.6-21.4$ & 21.5 & 5 \\
\hline & & & & & & &
\end{tabular}

TABLE 3: HTER values for MLP at EER working point.

\begin{tabular}{ccccc}
\hline & & \multicolumn{3}{c}{ MLP } \\
& & FAR-FRR & HTER & $n$ \\
\hline \multirow{3}{*}{ CdB } & CbCr & $7.5-9.7$ & 8.6 & 20 \\
& $\mathbf{a}^{*} \mathbf{b}^{*}$ & $5.3-5.7$ & 5.5 & 5 \\
& $\mathbf{r g}$ & $6.8-5.9$ & 6.3 & 15 \\
\hline \multirow{3}{*}{ UdB } & $\mathbf{C b C r}$ & $9.5-13.1$ & 11.3 & 10 \\
& $\mathbf{a}^{*} \mathbf{b}^{*}$ & $11.0-13.3$ & 12.1 & 10 \\
& $\mathbf{r g}$ & $7.6-15.6$ & 11.6 & 5 \\
\hline
\end{tabular}

When the number of samples used for adjusting the GMM model decreases from 90,000 to 250 (the same number used for training the SVM models), the performance in terms of HTER is similar, but the EER threshold (that uses non skin samples) was clearly more robust if more samples were used to estimate it, that is, by using 250 samples, the difficulty of generalizing an EER point increases. For example, in $\mathrm{CbCr}$ color space, FAR $=18.1, \mathrm{FRR}=29.0$ by using 250 samples and $\mathrm{FAR}=24.0, \mathrm{FRR}=23.8$ with 90,000 samples.

Table 3 shows the results for MLP with one hidden layer and $n$ hidden neurons. Similarly to GMM, performance for $\mathrm{CdB}$ is better than for UdB in the three color spaces, but the network complexity, measured as the optimal number of hidden neurons, is higher in $\mathrm{CbCr}$ and $\mathrm{rg}$ for $\mathrm{CdB}$ than for UdB. Therefore, under light intensity uncontrolled conditions, the performance does not improve by using more complex networks. Moreover, note that each color space in each database requires a different network complexity. Comparing the values of HTER with the corresponding ones obtained with GMM, MLP is superior to GMM in all considered cases. This improvement is even higher for UdB.

4.4. Results with Kernel-Based Segmentation. As described in Section 2, an SVM and two SVND algorithms (SVND-H and SVND-S) have been considered. For all of them, model tuning must be first addressed, and the free parameters of the model $(\{C, \sigma\}$ in SVM and SVND-S, and $\{\nu, \sigma\}$ in SVND$\mathrm{H})$ have to be properly tuned. Recall that both $C$ and $v$ are introduced to balance the margin and the losses in their respective problems, whereas $\sigma$ represents in both cases the width of the Gaussian kernel. Therefore, these parameters are expected to be dependent on the training data.
The training and the test subsets were obtained from two main considerations. First, although the SVMs can be trained with large and high-dimensional training sets, it is also well known that the computational cost increases when the optimal model parameters are obtained by using the classical Quadratic Programing as optimization method. And second, the SVMs methods have shown a good generalization capability for a lot of different problems previously in literature. Due to both reasons, a total of only 250 skin samples were randomly picked (from the GMM training set) for the two SVND algorithms, and a total of only 500 samples (the previous 250 skin samples plus 250 non-skin samples randomly picked from the GMM tuning set) for the SVM model.

After considering enough wide ranges to ensure that both optimal free parameters of each SVM model $(\{C, \sigma\}$ for SVND-S and SVM; $\{\nu, \sigma\}$ for SVND-H) can be obtained, we found that with SVND-S, $\{C=0.5, \sigma=0.05\}$ were selected as the optimal values of the free parameters for the three color spaces and $\mathrm{CdB}$ database, and $\{C=0.05, \sigma=0.1\}$ for the three color spaces and UdB database; with SVND-H, the most appropiate values for the three color spaces were $\{v=$ $0.01, \sigma=0.05\}$ for $\mathrm{CdB}$ database, and $\{\nu=0.08, \sigma=0.2\}$ for UdB; and with SVM, the optimal values for all color spaces were $\{C=46.4, \sigma=1.5\}$ for $\mathrm{CdB}$ and $\{C=215.4, \sigma=2.5\}$ for UdB.

Table 4 shows the detailed results for three kernel methods: SVND-H, SVND-S, and SVM, with their free parameters. The performance obtained with both SVND methods is very similar, as HTER and MC values are very close for the same color space and the same database. Although the lowest values of HTER are achieved with SVM in all the cases, the improvement is even higher for UdB. For example, in rg color space and CdB, HTER $=5.8$ with SVM versus HTER $=6.4$ with SVDN mehods, while for UdB, HTER $=10.8$ with SVM and HTER $>13$ with SVDN. When we focus on the performance in terms of EER threshold, the behaviour of SVND methods shows more robustness, that is, the FAR and FRR values are closer than those achieved with SVM. Moreover, although the SVM gets the lowest HTER values for $\mathrm{Cdb}$ and $\mathrm{UdB}$, the required complexity for $\mathrm{UdB}$, measured in terms of MC values, is higher than the corresponding one required by SVND methods (from $\mathrm{MC}=23.6$ with SVM to MC $=5.6$ with SVND-S and SVND-H).

4.5. Comparison of Methods. As an example, Figure 4 shows the training samples and boundaries obtained with nonparametric detectors (SVND-H, SVND-S, SVM, and MLP), and for the three color spaces and both databases (CdB and UdB). Note that in the two SVND algorithms, the boundaries in terms of EER, obtained with the tuning set, were very close to those given by the algorithm boundary: $R_{0}$ for SVND-S and $\rho_{0}$ for SVND-H. Accordingly, a good first estimation of the EER boundary can be done just by considering only the skin samples of the training set, thus avoiding the selection of an EER threshold over a tuning set. Therefore, no subset of nonskin samples is needed with SVND for building a complete 


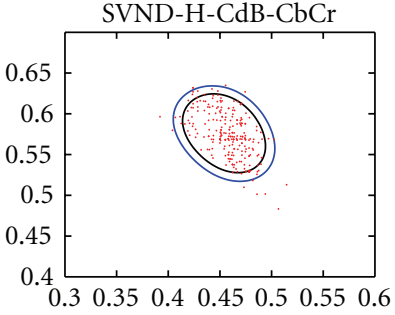

(a0)

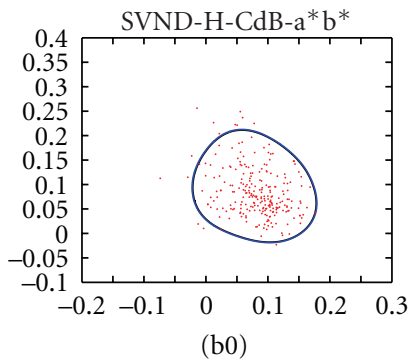

SVND-H-CdB-rg

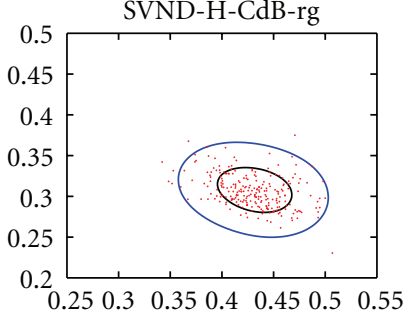

(c0)

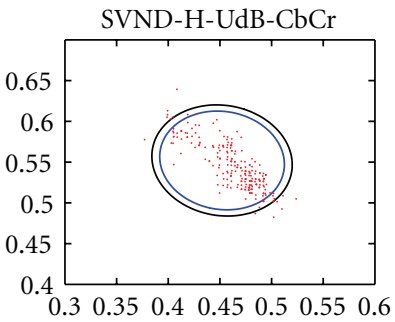

(d0)

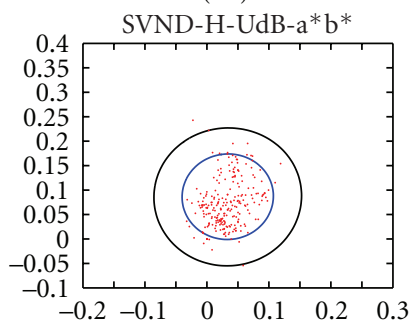

(e0)

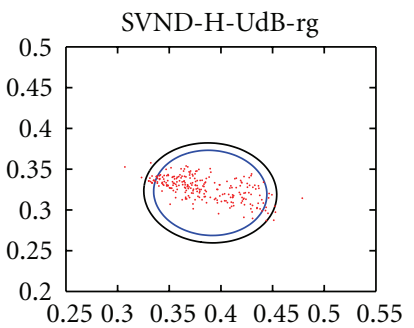

(f0)

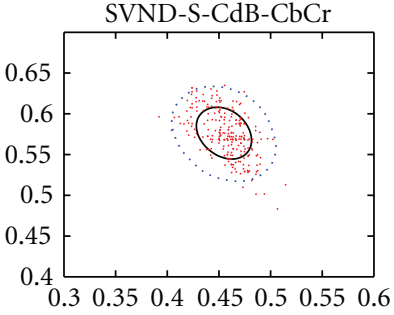

(a1)

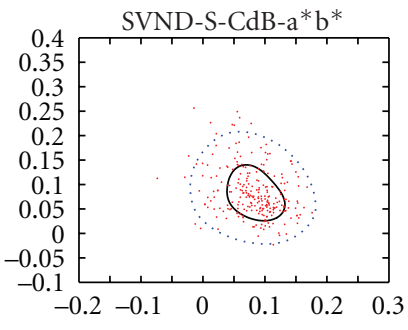

(b1)

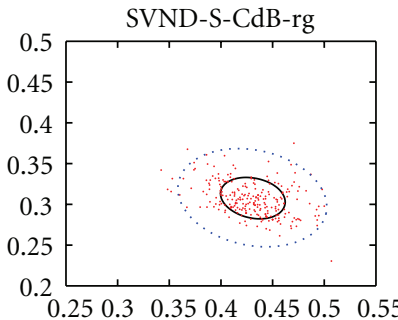

(c1)

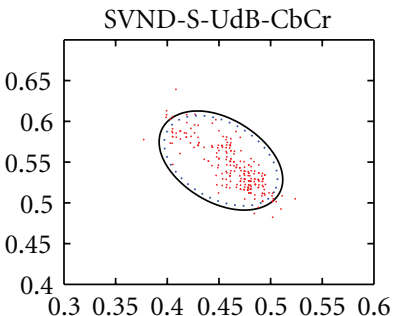

(d1)

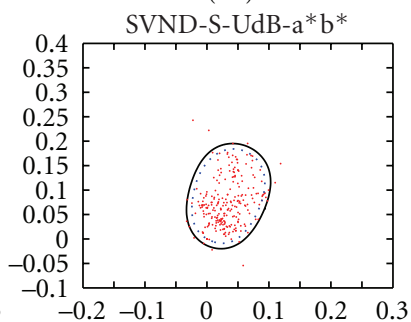

(e1)

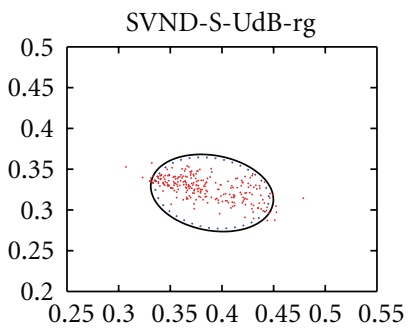

(f1)

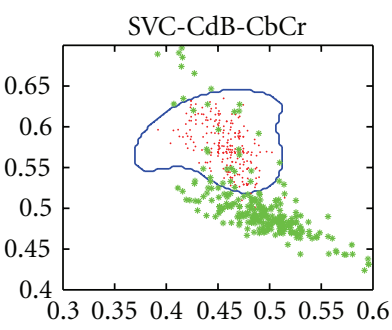

(a2)

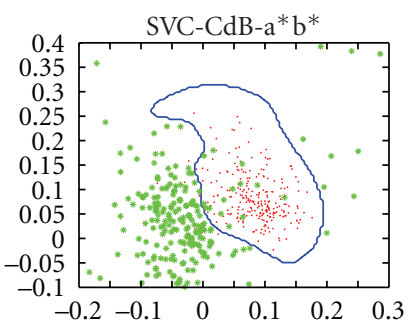

(b2)

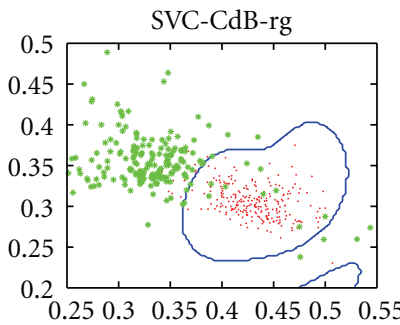

(c2)

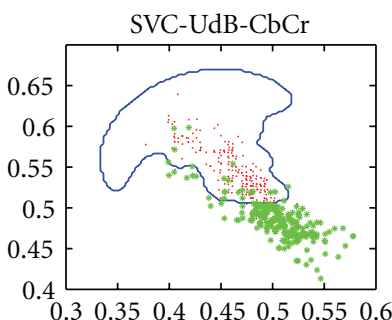

(d2)

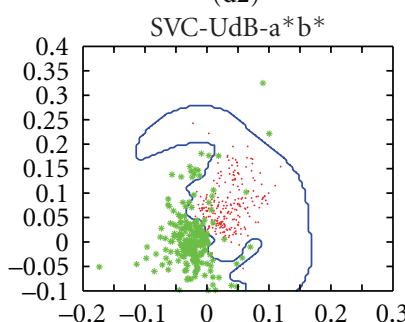

(e2)

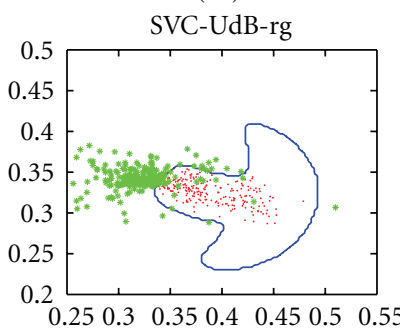

(f2)

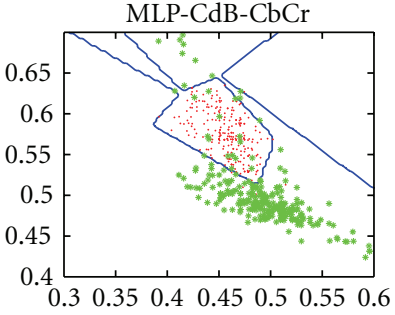

(a3)

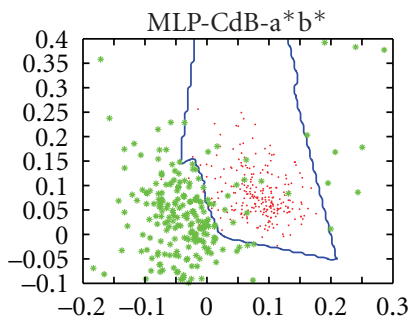

(b3)

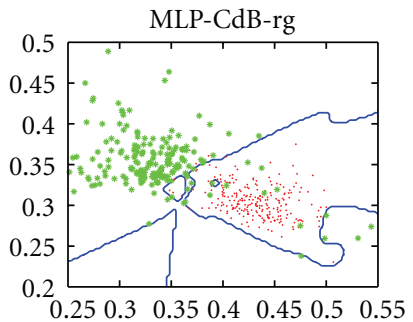

(c3)

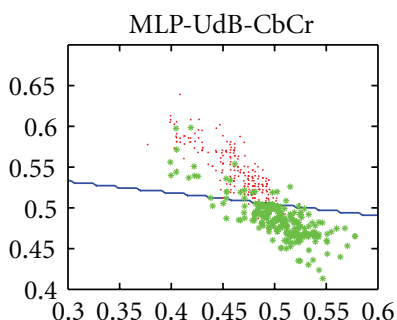

(d3)

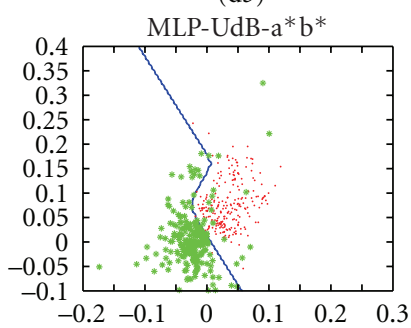

(e3)

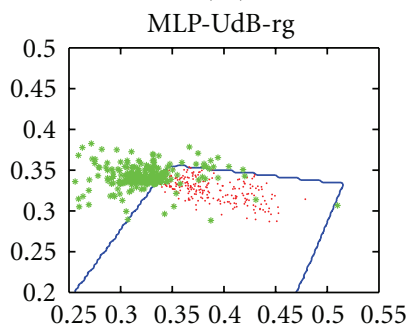

(f3)

FIgURE 4: Training samples (skin in red, nonskin in green) and skin boundaries (continuous for SVND threshold, dashed for EER threshold), obtained from the nonparametric models (each column corresponds to a model: SVND-H in $* 0$, SVND-S in $* 1$, SVM in $* 2$, and MLP in $* 3)$. $\mathrm{CdB}$ with $\mathrm{CbCr}$ in $a *, \mathrm{CdB}$ with $\mathrm{a}^{\star} \mathrm{b}^{\star}$ in $b *, \mathrm{CdB}$ with $\mathrm{rg}$ in $c *, \mathrm{UdB}$ with $\mathrm{CbCr}$ in $d *$, $\mathrm{UdB}$ with $\mathrm{a}^{\star} \mathrm{b}^{\star}$ in $e *, \mathrm{UdB}$ with $\mathrm{rg}$ in $f *$. 
TABLE 4: Values of HTER (\%) and complexity for SVND-H $(n u=0.01, \sigma=0.05$ for CdB; $n u=0.08, \sigma=0.2$ for UdB $)$, SVND-S $(C=0.5$, $\sigma=0.05$ for $\mathrm{CdB} ; C=0.05, \sigma=0.1$ for $\mathrm{UdB})$ and $\operatorname{SVM}(C=46.4, \sigma=1.5$ for $\mathrm{CdB} ; C=215.4, \sigma=2.5$ for $\mathrm{UdB})$.

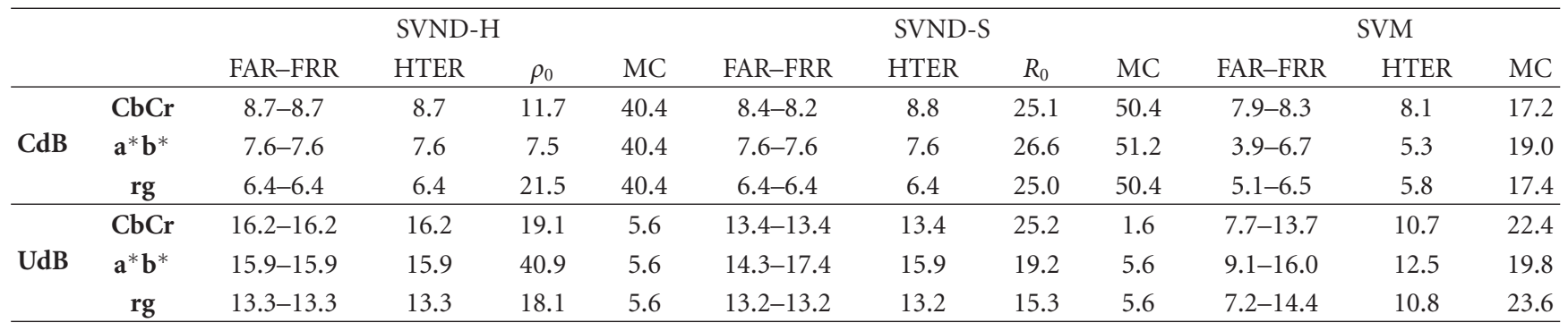

TABLE 5: All values of HTER (\%).

\begin{tabular}{ccccccc}
\hline & & SVND-H & SVND-S & SVM & MLP & GMM \\
\hline \multirow{4}{*}{$\mathbf{C d B}$} & $\mathbf{C b C r}$ & 8.7 & 8.8 & 8.1 & 8.6 & 11.3 \\
& $\mathbf{a}^{*} \mathbf{b}^{*}$ & 7.6 & 7.6 & 5.3 & 5.5 & 7.1 \\
& $\mathbf{r g}$ & 6.4 & 6.4 & 5.8 & 6.3 & 7.4 \\
\hline \multirow{4}{*}{$\mathbf{U d B}$} & $\mathbf{C b C r}$ & 16.2 & 13.4 & 10.7 & 11.3 & 23.6 \\
& $\mathbf{a}^{*} \mathbf{b}^{*}$ & 15.9 & 15.9 & 12.5 & 12.1 & 22.6 \\
& $\mathbf{r g}$ & 13.3 & 13.2 & 10.8 & 11.6 & 21.8 \\
\hline
\end{tabular}

TABLE 6: HTER values at EER for two-class SVM and 3D color spaces.

\begin{tabular}{ccccc}
\hline & & \multicolumn{3}{c}{ SVM } \\
& & FAR-FRR & HTER & MC \\
\hline \multirow{3}{*}{ CdB } & YCbCr & $6.7-4.9$ & 5.8 & 16 \\
& CIEL $^{*} \mathbf{a}^{*} \mathbf{b}^{*}$ & $4.6-6.7$ & 5.6 & 22 \\
& rgb & $5.8-6.7$ & 6.2 & 19 \\
\hline \multirow{3}{*}{ UdB } & YCbCr & $6.9-21.5$ & 14.2 & 24.8 \\
& CIEL $^{*} \mathbf{a}^{*} \mathbf{b}^{*}$ & $7.0-23.5$ & 15.2 & 23.2 \\
& $\mathbf{r g b}$ & $7.4-14.3$ & 10.8 & 25.6 \\
\hline
\end{tabular}

skin detector, though the use of a test set with samples from both classes can be useful for a subsequent security verification of the threshold provided by the algorithm. Nevertheless, due to the extremely high density of samples near the decision boundaries, those nonparametric models trained with skin and non-skin samples are able to yield more complex and accurate boundaries, whereas models trained with only skin samples yield a good skin domain description at the expense of increased skin and non-skin samples overlapping. The effect of the boundary estimation on the segmentation can be seen in Figure 4, which shows several representative examples of the pixel-classified images in $\mathrm{CdB}$ and $\mathrm{UdB}$ by using the analyzed detectors.

A summary of the performance obtained by the five different classifier (in terms of HTER over the test data set) can be found in Table 5. We can conclude that, under controlled image acquisition conditions, nonparametric methods yield higher accuracy than GMM. The difference is even higher under uncontrolled capturing conditions. For example, with $\mathrm{a}^{\star} \mathrm{b}^{\star}$ color space in UdB, HTER $=22.6$ for GMM versus HTER $=15.9$ for SVND-H (in this case, the worse of the three SVM-based methods considered). It is interesting to emphasize that both SVND models can be also seen as isotropic Gaussian mixtures (see (17) and (27)), with the important difference that SVND training puts the centers of Gaussian kernels at samples (support vectors) that are more relevant for describing the domain of interest. We must remark also that SVM-based segmentation algorithms are nonparametric methods which obtain the required $\mathrm{MC}$ from the available data, thus avoiding searches like the number of components in GMM. When comparing kernel-based methods with MLP, the last one shows lower HTER values than GMM and SVNN for most of the color spaces, but always higher than the corresponding ones of SVM (the differences are significant according to a paired-sample Ttest). Therefore, the MLP can be considered as an alternative to SVDN methods, but not to SVM. Moreover, MLP has the problem of finding local minimum solutions, while SVM always finds the global minimum.

With respect to the SVM-based methods, we can conclude that the best performance, in terms of HTER, is provided by the standard SVM classifier for all the color spaces and databases studied. Hence, when the goal of the application under study is the skin segmentation, this is a more appropriate approach to be considered. However, when it is pursued to obtain an adequate description of the domain that represents the support for skin pixels in the color space, rather than its statistical density descriptions, the best solution is to use an SVND algorithm. Moreover, with SVND algorithms, $R_{0}$ and $\rho_{0}$ values can be considered as default decision statistics or thresholds, for SVND-S and SVND-H, respectively, while for GMM and SVM the decision statistic must be set a posteriori and non-skin samples are required.

4.6. Two-Class SVM and 3D Color Spaces. As we mentioned in Section 2.1, we have constrained our experiments to the application cases where not enough 3D labeled data is available for an accurate modeling of the $3 \mathrm{D}$ color space. In order to show that the skin segmentation performs better in this application if only 2D color spaces are considered, we have obtained the performance for the two-class SVM classifier (the best of the five considered for 2D color spaces) in the three different 3D color spaces and the two databases, by considering the same conditions (500 training samples). The obtained results are shown in Table 6, which shows that the HTER values are higher than the corresponding ones obtained by using only $2 \mathrm{D}$ spaces, except for $\mathrm{YCbCr}-\mathrm{CdB}$ (see Table 4). Moreover, the differences are higher under uncontrolled lighting conditions. 


\section{Conclusions}

We have presented a comparative study between pixel-wise skin color detection using GMM, MLP and a three different kernel-based methods: the classical SVM, and two oneclass methods (SVND) on three different chromaticity color spaces. All kernel-based models studied have shown some interesting advantages for skin detection applications when compared to GMM and MLP. Moreover, each SVM-based method solves a QP problem, which has a unique solution, and hence there is no randomness in the initialization settings. When the main interest of the application is an adequate description of the skin pixel domain, the SVND approaches have shown to be more adequate than those based on modeling probability density function. However, when the objective is the skin detection, which is a more usual application in practice, the classical SVM outperformed the SVND ones in terms of HTER for the three color spaces and the two different databases (under controlled and specially under uncontrolled lighting conditions) considered, due to its use of the boundary information from skin and non-skin samples during its design. Our aim was to focus on two characteristics of the broad skin segmentation problem, namely, the importance of controlled lighting and acquisition conditions, and the influence of the chromaticity color spaces. In this work we have created our dataset with only caucasian people; the extension to schemes dealing with other-skin tones is one of the main related future research issues.

\section{Acknowlegment}

This work has been partially supported by Research Projects TEC2007-68096-C02/TCM and TEC2008-05894 from Spanish Government.

\section{References}

[1] J. Cai, A. Goshtasby, and C. Yu, "Detecting human faces in color images," Image and Vision Computing, vol. 18, no. 1, pp. 63-75, 1999.

[2] R.-L. Hsu, M. Abdel-Mottaleb, and A. K. Jain, "Face detection in color images," IEEE Transactions on Pattern Analysis and Machine Intelligence, vol. 24, no. 5, pp. 696-706, 2002.

[3] M. H. Yang and N. Ahuja, "Extracting gestural motion trajectory," in Proceedings of the 3rd IEEE International Conference on Automatic Face and Gesture Recognition, 1998.

[4] K.-K. Sung and T. Poggio, "Example-based learning for viewbased human face detection," IEEE Transactions on Pattern Analysis and Machine Intelligence, vol. 20, no. 1, pp. 39-51, 1998.

[5] Y. Li, A. Goshtasby, and O. Garcia, "Detecting and tracking human faces in videos," in Proceedings of the 15th IEEE International Conference on Pattern Recognition (ICPR '00), vol. 1, pp. 807-810, 2000.

[6] M.-J. Chen, M.-C. Chi, C.-T. Hsu, and J.-W. Chen, "ROI video coding based on H.263+ with robust skin-color detection technique," IEEE Transactions on Consumer Electronics, vol. 49, no. 3, pp. 724-730, 2003.
[7] J. Brand and J. S. Mason, "A comparative assessment of three approaches to pixel-level human skin-detection," in Proceedings of the 15th IEEE International Conference on Pattern Recognition ((ICPR '00), vol. 1, pp. 1056-1059, 2000.

[8] H. Wang and S.-F. Chang, "A highly efficient system for automatic face region detection in MPEG video," IEEE Transactions on Circuits and Systems for Video Technology, vol. 7, no. 4, pp. 615-628, 1997.

[9] M.-H. Yang and N. Ahuja, "Detecting human faces in color images," in Proceedings of the IEEE International Conference on Image Processing, vol. 1, pp. 127-130, 1998.

[10] J. C. Terrillon, M. N. Shirazi, H. Fukamachi, and S. Akamatsu, "Comparative performance of different skin chrominance models and chrominance spaces for the automatic detection of human faces in color images," in Proceedings of the 5th IEEE International Conference on Automatic Face and Gesture Recognition, 2000.

[11] M.-H. Yang and N. Ahuja, "Gaussian mixture model for human skin color and its applications in image and video databases," in Conference on Storage and Retrieval for Image and Video Databases, vol. 3656 of Proceedings of SPIE, pp. 458466, 1999.

[12] S. L. Phung, A. Bouzerdoum, and D. Chai, "Skin segmentation using color pixel classification: analysis and comparison," IEEE Transactions on Pattern Analysis and Machine Intelligence, vol. 27, no. 1, pp. 148-154, 2005.

[13] M. J. Jones and J. M. Rehg, "Statistical color models with application to skin detection," International Journal of Computer Vision, vol. 46, no. 1, pp. 81-96, 2002.

[14] H. Jin, Q. Liu, H. Lu, and X. Tong, "Face detection using oneclass SVM in color images," in Proceedings of the International Conference on Signal Processing (ICSP '04), pp. 1432-1435, 2004.

[15] R. N. Hota, V. Venkoparao, and S. Bedros, "Face detection by using skin color model based on one class classifier," in Proceedings of the 9th International Conference on Information Technology (ICIT '06), pp. 15-16, 2006.

[16] J.-C. Terrillon, M. N. Shirazi, M. Sadek, H. Fukamachi, and T. S. Akamatsu, "Invariant face detection with support vector machines," in Proceedings of the 15th IEEE International Conference on Pattern Recognition (ICPR '00), 2000.

[17] Z. Xu and M. Zhu, "Color-based skin detection: survey and evaluation," in Proceedings of the 12th International MultiMedia Modelling Conference (MMM '06), pp. 143-152, 2006.

[18] B. Schölkopf, R. C. Williamson, A. J. Smola, J. Shawe-Taylor, and J. Platt, "Support vector method for novelty detection," in Advances in Neural Information Processing Systems, vol. 12, 2000.

[19] D. M. J. Tax and R. P. W. Duin, "Support vector domain description," Pattern Recognition Letters, vol. 20, no. 11-13, pp. 1191-1199, 1999.

[20] B. D. Zarit, B. J. Super, and F. H. Queck, "Comparison of five color models in skin pixel classification," in Proceedings of the International Workshop on Recognition, Analysis, and Tracking of Faces and Gestures in Real-Time Systems, 1999.

[21] A. Albiol, L. Torres, and E. J. Delp, "Optimum color spaces for skin detection," in Proceedings of the IEEE International Conference on Image Processing, vol. 1, pp. 122-124, 2001.

[22] S. Jayaram, S. Schmugge, M. C. Shin, and L. V. Tsap, "Effect of colorspace transformation, the illuminance component, and color modeling on skin detection," in Proceedings of the IEEE Computer Society Conference on Computer Vision and Pattern Recognition (CVPR '04), vol. 2, pp. 813-818, 2004. 
[23] M. Soriano, B. Martinkauppi, S. Huovinen, and M. Laaksonen, "Adaptive skin color modeling using the skin locus for selecting training pixels," Pattern Recognition, vol. 36, no. 3, pp. 681-690, 2003.

[24] A. Dempster, N. Laird, and D. Rubin, "Maximum likelihood from incomplete data via the EM algorithm," Journal of the Royal Statistical Society, Series B, vol. 39, no. 1, pp. 1-38, 1997.

[25] G. Camps-Valls, J. L. Rojo-Álvarez, and M. Martínez-Ramón, Kernel Methods in Bioengineering, Communications and Image Processing, IDEA Group, 2006.

[26] V. Vapnik, Statistical Learning Theory, John Wiley \& Sons, New York, NY, USA, 1998.

[27] K. Messer, J. Matas, J. Kittler, J. Luettin, and G. Maitre, "Xm2vtsdb: the extended m2vts database," in Proceedings of the International Conference on Audioand Video-Based Biometric Person Authentication (AVBPA '99), 1999.

[28] E. Bailly-Bailliére, S. Bengio, F. Bimbot, et al., "The BANCA database and evaluation protocol," in Proceedings of the 4th International Conference on Audioand Video-Based Biometric Person Authentication (AVBPA '03), pp. 625-638, 2003.

[29] B. Menser and M. Brunig, "Locating human faces in color images with complex background," in Proceedings of the IEEE International Symposium on Intelligent Signal Processing and Communication Systems (ISPACS '99), pp. 533-536, 1999.

[30] K. Sobottka and I. Pitas, "A novel method for automatic face segmentation, facial feature extraction and tracking," Signal Processing: Image Communication, vol. 12, no. 3, pp. 263-281, 1998. 